\title{
The neurophysiological lesson from the Italian CIDP database
}

\author{
Emanuele Spina ${ }^{1}$ (D) $\cdot$ Pietro Emiliano Doneddu ${ }^{2} \cdot$ Giuseppe Liberatore $^{2} \cdot$ Dario Cocito $^{3} \cdot$ Raffaella Fazio $^{4}$. \\ Chiara Briani ${ }^{5}$. Massimiliano Filosto ${ }^{6}$. Luana Benedetti ${ }^{7,8}$. Giovanni Antonini ${ }^{9}$. Giuseppe Cosentino ${ }^{10,11}$. \\ Stefano Jann ${ }^{12}$. Anna Mazzeo ${ }^{13}$ - Andrea Cortese ${ }^{11,14}$. Girolama Alessandra Marfia ${ }^{15}$. Angelo Maurizio Clerici ${ }^{16}$. \\ Gabriele Siciliano ${ }^{17}$. Marinella Carpo ${ }^{18} \cdot$ Marco Luigetti $^{19} \cdot$ Giuseppe Lauria $^{20,21} \cdot$ Tiziana Rosso $^{22} \cdot$ Guido Cavaletti $^{23}$. \\ Erdita Peci ${ }^{3}$ - Stefano Tronci ${ }^{4}$ - Marta Ruiz ${ }^{5}$ - Stefano Cotti Piccinelli ${ }^{6}$. Angelo Schenone ${ }^{7}$ - Luca Leonardi ${ }^{9}$.

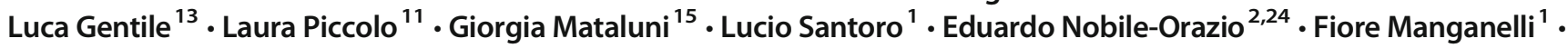 \\ on the behalf of the Italian CIDP Database Study Group
}

Received: 28 January 2021 / Accepted: 14 May 2021 / Published online: 21 May 2021

(C) The Author(s) 2021

\begin{abstract}
Introduction Electrophysiological diagnosis of chronic inflammatory demyelinating polyradiculoneuropathy (CIDP) may be challenging. Thus, with the aim ofproviding some practical advice in electrophysiological approach to a patient with suspected CIDP, we analyzed electrophysiological data from 499 patients enrolled inthe Italian CIDP Database.

Methods We calculated the rate of each demyelinating feature, the rate of demyelinating features per nerve, the diagnostic rate for upper andlower limb nerves, and, using a ROC curve analysis, the diagnostic accuracy of each couple of nerves and each demyelinating feature, for every CIDP subtype.Moreover, we compared the electrophysiological data of definite and probable CIDP patients with those of possible and not-fulfilling CIDP patients, and by a logisticregression analysis, we estimated the odds ratio (OR) to make an electrophysiological diagnosis of definite or probable CIDP.

Results The ulnar nerve had the highestrate of demyelinating features and, when tested bilaterally, had the highest diagnostic accuracy except for DADS in which peroneal nerves were the most informative.In possible and not-fulfilling CIDP patients, a lower number of nerves and proximal temporal dispersion (TD) measurements had been performed compared to definiteand probable CIDP patients. Importantly, OR for each tested motor nerve and each TD measurement was 1.59 and 1.33, respectively. Conclusion Our findingsdemonstrated that the diagnosis of CIDP may be missed due to inadequate or incomplete electrophysiological examination or interpretation. At the same time, thesedata taken together could be useful to draw a thoughtful electrophysiological approach to patients suspected of CIDP.
\end{abstract}

Keywords CIDP $\cdot$ Neurophysiology $\cdot$ Diagnostic criteria $\cdot$ Nerve conduction

\section{Introduction}

Chronic inflammatory demyelinating polyradiculoneuropathy (CIDP) is a chronic and disabling disease with a range prevalence of $0.8-8.9$ cases per 100,000 and incidence of $0.2-1.6$ / 100,000 [1]. It is the most frequent acquired immunemediated chronic neuropathy and has a broad spectrum of

Emanuele Spina ema.spina@libero.it

Fiore Manganelli

fioremanganelli@gmail.com

Extended author information available on the last page of the article possible clinical presentation including typical and atypical forms [2-4].

Diagnosis of CIDP is made by demonstrating peripheral nerve demyelination, commonly by electrophysiological testing. Hence, several sets of electrophysiological criteria for diagnosis of CIDP have been proposed, and the most widely accepted criteria are those recommended by the European Federation of Neurological Societies and Peripheral Nerve Society (EFNS/PNS). Based on the number and electrophysiological features (i.e., prolonged distal motor latency (DML), motor nerve conduction velocity (MNCV) slowing, temporal dispersion (TD), probable or definite conduction block (CB), and absent or prolonged $\mathrm{F}$-wave), three diagnostic electrophysiological classes are identifiable: definite, probable, and possible CIDP [5-7]. 
The diagnosis may be easy when demyelinating signs are widespread, while it may be challenging when demyelination is limited to few or specific nerves or nervous tracts (e.g., proximally) [8-10].

Accordingly, in clinical practice, neurophysiological examination in search of demyelinating signs may be timeconsuming and eventually not decisive, so making tricky the diagnosis and delaying the start of a proper treatment.

Thus, having the opportunity to access the Italian CIDP Database [3, 4], we have analyzed a large amount of electrophysiological data. Our aim was to obtain a wide overview of the electrophysiological characteristics of CIDP that could provide some practical advice in electrophysiological approach to a patient with suspected CIDP.

Moreover, we analyzed the electrophysiological data from patients who, although diagnosed as having CIDP based on clinical and supportive features, did not fulfill the electrophysiological criteria for demyelination.

\section{Patients and methods}

This was a retrospective multicenter cohort study in a large sample of CIDP patients implementing a web database (CINECA, Bologna, Italy) to collect demographical, clinical, and electrophysiological data from patients diagnosed and followed by 22 centers throughout Italy with expertise on CIDP.

The ethical committee of each participating center approved the study and all the patients gave written informed consent.

Clinical and neurophysiological data were obtained by an experienced neurologist with a neuromuscular subspecialty. Verification of the diagnostic data for all of the enrolled patients was centralized in the database coordinator center (Humanitas). Data monitoring included diagnosis revision, suspect double entries, missing data, and plausibility checks. Patients with an alternative diagnosis for the neuropathy, increased titers of anti-myelin-associated glycoprotein IgM antibodies, or without available nerve conduction studies were excluded [11].

For the current study, all electrophysiological data (blinded compared to clinical and laboratory data) were further evaluated independently and after discussed all together by three expert neurophysiologists (ES, LS, FM) in the center of Naples. Based on EFNS/PNS criteria for demyelination, patients were classified into four diagnostic categories: "definite," "probable," "possible," and "not-fulfilling" electrophysiological diagnosis of CIDP. Afterward, the electrophysiological results were discussed with the coordinating center (ENO, PD) and combined with clinical and laboratory data. The neurophysiological study was contemporaneous with clinical classification of CIDP.
We calculated in CIDP patients (definite, probable, and possible) the rate of each demyelinating feature, the rate of demyelinating features per nerve (median, ulnar, peroneal, and tibial), and the diagnostic rate as established by EFNS/PNS for upper and lower limb nerves. To exclude median neuropathy at the wrist (carpal tunnel syndrome), distal motor latency in the median nerve was not considered in our analysis when associated with slowing of sensory nerve conduction velocities or absence of sensory action potential.

We ran a receiver operating characteristic (ROC) curve analysis to measure the diagnostic accuracy for each possible couple of nerves and for each demyelinating feature for CIDP diagnosis (definite, probable, or possible). For ROC curve analysis, we used as diagnostic gold standard the diagnosis of CIDP based on the combination of clinical features, neurophysiology data, and supportive criteria.

We also ran ROC curve analysis separately for typical CIDP and for each atypical CIDP subtype (i.e., multifocal acquired demyelinating sensory and motor (MADSAM), distal acquired demyelinating symmetric (DADS), pure sensory ,and pure motor). Values for the area under the curve (AUC) obtained from ROC curve analysis above 0.8 were considered excellent [12].

Moreover, we compared the electrophysiological approach (i.e., number of tested motor nerves and number of each electrophysiological measure per patient) among the patients with definite and probable CIDP and those with possible CIDP and not fulfilling electrophysiological CIDP criteria. We decided to analyze together possible and not-fulfilling CIDP patients as "possible" category is a very poorly specific category [5].

Thus, through a logistic regression analysis, we estimated the odds ratio (OR) of differences in electrophysiological approach applied to patients with definite and probable CIDP with respect to that applied to possible and not-fulfilling groups and then we used a multivariable logistic regression analysis for estimating the independent weight of differences emerged from the univariate logistic regression analysis.

Lastly, we evaluated the percentage of diagnosed patients (definite and probable) according to the number of tested nerves $(2,3,4,5,6,7$, and 8 nerves $)$ and the number of proximal temporal dispersion measurements. After finding that the percentage of CIDP diagnosis progressively increased up to six tested nerves while after (more than six nerves) the diagnostic rate remained unchanged, the value of 6 (nerves) was set as a cut-off and as a reference value for a regression analysis.

Each regression analysis was controlled for age and sex, and odds ratios and $95 \%$ confidence intervals were reported.

\section{Ethics approval}

The study was approved by the ethical committee of each participating center. 


\section{Consent to participate}

Written informed consent was obtained from all participants at enrollment.

\section{Data availability Policy}

Any data not published within this article is fully available previous anonymization by request from a qualified investigator.

\section{Results}

We analyzed data from 499 (out of 545) patients included consecutively into the Italian CIDP Database from January 2015 to January 2019. Forty-four patients were excluded from the analysis for the presence of a different cause for neuropathy or unavailable neurophysiological data [11]. Two patients with chronic immune sensory polyradiculopathy (CISP) were excluded from the analysis [13].

First, we divided our dataset according to electrophysiological data: $352 / 499$ patients (70.6\%) were classified as definite CIDP, $10 / 499$ (2\%) as probable CIDP, $57 / 499(11.4 \%)$ as possible CIDP, and 80/499 (16\%) as CIDP not fulfilling the EFNS/PNS electrophysiological criteria [6]. Then, we evaluated the subtype: typical patients were $397 / 499$ (79.7\%); MADSAM 18/499 (3.4\%), DADS 42/499 (8.4\%), 20/499 pure motor (4\%), 17/499 pure sensory (3.4\%), and 5/499 focal (1\%) (Supplementary Table 1).

For patients that did not meet EFNS/PNS electrophysiological criteria for demyelination, the diagnosis of CIDP was discussed among the neurophysiologist panel, the database coordinator center, and the physician who had enrolled the patient. Therefore, these patients were considered affected by CIDP because, though they did not have nerve conduction abnormalities (Supplementary Table 2), satisfying EFNS/PNS criteria, nevertheless, they showed clinical, laboratory, and neurophysiological features (i.e., clinical relapse, response to therapies, elevated cerebrospinal fluid proteins, magnetic resonance imaging with gadolinium enhancement, nerve biopsy, somatosensory-evoked potentials) supportive of CIDP.

In definite, probable, and possible CIDP patients as a whole, DML was prolonged in 160/419 (38.2\%), MNCV was reduced in 285/419 (68\%), proximal TD was present in $183 / 419$ (43.7\%), probable and definite CBs were observed in 46/419 (11\%) and 134/419 (32\%), respectively, and F-wave was absent or prolonged in 145/419 (34.6\%) patients. Prolonged distal compound muscle action potential (CMAP) duration was excluded from this analysis because of heterogeneity of data.

The highest rate of demyelinating features was observed in the ulnar nerve $($ right $=67.9 \%$; left $=69.3 \%)$. The frequency for each demyelinating feature per nerve (median, ulnar, peroneal, and tibial) is reported in Table 1.

Overall, at least one nerve with demyelinating features was observed in $65.7 \%$ of patients considering only the upper limbs, and in $64.5 \%$ of patients considering only the lower limbs. However, the analysis per subtypes showed that the rate of demyelinating features was comparable between upper and lower limbs for typical and sensory forms while it was higher in upper than lower limbs in MADSAM (70.6 vs $58.9 \%)$ and motor $(64.7 \%$ vs $35.3 \%)$ subtypes and it was higher in lower limbs in DADS phenotype $(73.8 \%$ vs $40.5 \%$ ) (Table 2).

The ROC curve analysis for nerves showed the highest diagnostic accuracy for ulnar nerves and the lowest for tibial nerves (Table 3 and Fig. 1) in the overall population.

In typical CIDP, as well in MADSAM, sensory, and motor subtypes, the ROC curve analysis showed the highest diagnostic accuracy for ulnar nerve evaluated bilaterally, followed by the combination of ulnar and peroneal nerves, independently from the side (Supplementary Table 3).

Instead, in DADS subtype, the highest diagnostic accuracy was observed for lower limb nerves and especially for peroneal nerve evaluated bilaterally, followed by the combination of peroneal and tibial (independently from the side). An excellent AUC was reported for the combination of ulnar and peroneal nerves, taken together (AUC: 0.95). Detailed data for each subtype are reported in Supplementary Table 4.

The ROC curve analysis for demyelinating features showed the highest diagnostic accuracy for MNCV slowing (AUC: 0.8401) (Fig. 2).

The patients with a diagnosis of definite and probable CIDP with respect to those with possible CIDP or not fulfilling electrophysiological CIDP criteria had a higher median value ( 6 vs 4 ) of tested motor nerves as a whole and also for upper (3 vs 2) and lower limbs (3 vs 2) considered separately.

In the entire cohort of patients, the median number of tested motor nerves was 6 (5.3 mean, 1.76 SD), 2 in the upper limbs (mean 2.6, 1.21 SD) and 3 in the lower limbs (mean 2.8, 1.1 $\mathrm{SD})$.

In definite CIDP patients, the median number of tested motor nerves was 6 (mean 5.6, SD 1.57), 3 in the upper limbs (mean 2.8, SD 1.13) and 3 in the lower limbs (mean 2.9, SD $1.1)$.

In probable CIDP patients, the median number of tested motor nerves was 6 (mean 5.4, SD 1.95), 3 in the upper limbs (2.9 mean, 1.1 SD) and 3 in the lower limbs (mean 2.5, 1.2 $\mathrm{SD})$.

In possible CIDP patients, the median number of tested motor nerves was 4 (4.5 mean, $2 \mathrm{SD}$ ), 2 in the upper limbs ( 2 mean, 1.2 SD) and in 2 in the lower limbs (mean 2.5, 1.2 $\mathrm{SD})$.

In CIDP patients not fulfilling the diagnostic criteria, the median number of tested motor nerves was 4 (4.6 mean, 1.8 


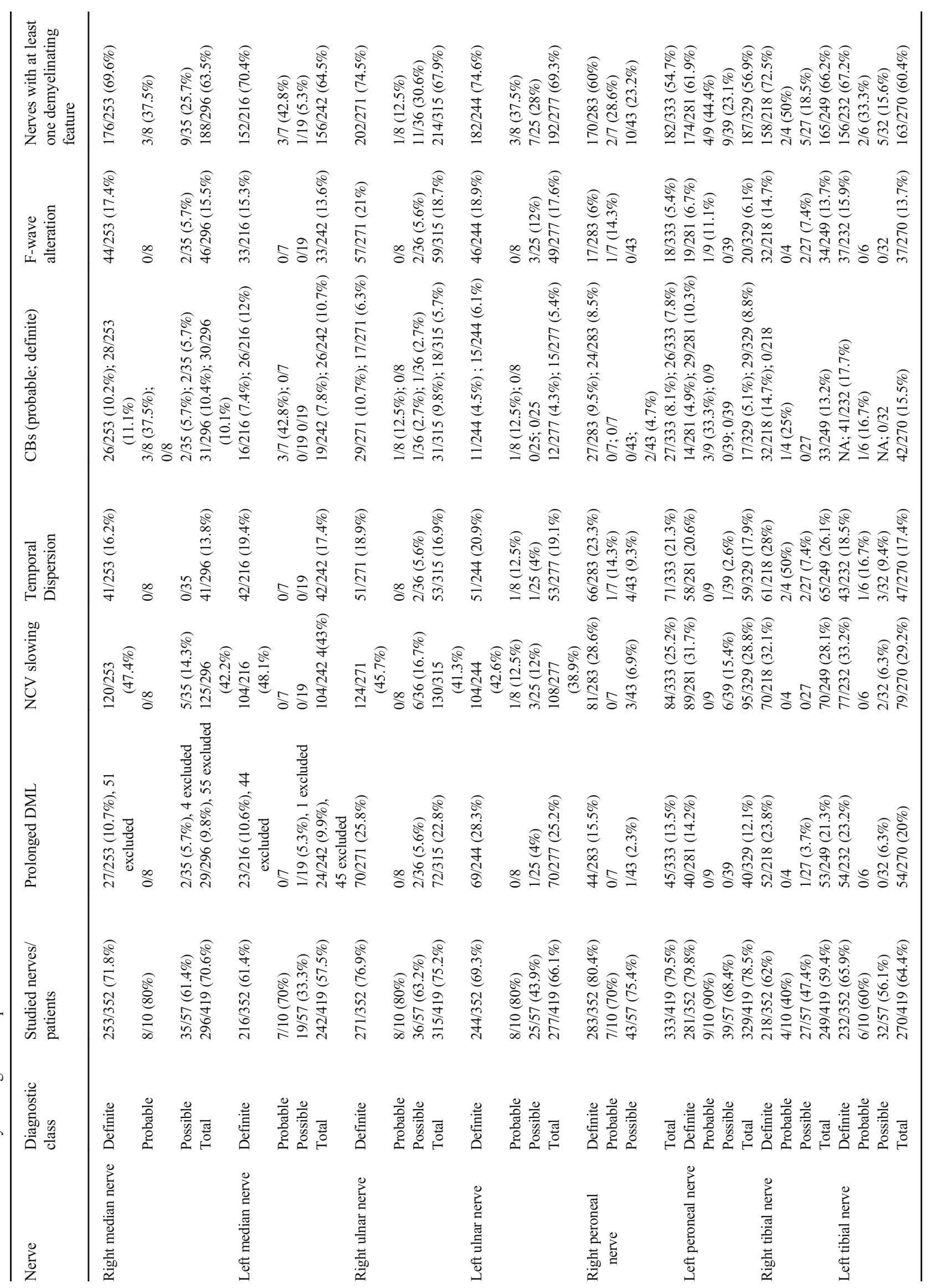


Table 2 Rate of patients with at least one demyelinating feature in upper and lower limbs

\begin{tabular}{|c|c|c|c|c|c|c|c|}
\hline & Overall & Typical & MADSAM & DADS & Sensory & Motor & Focal \\
\hline Upper limbs & $328 / 499(65.7 \%)$ & $275 / 397(69.3 \%)$ & $12 / 17(70.6 \%)$ & $17 / 42(40.5 \%)$ & $11 / 20(55 \%)$ & $11 / 17(64.7 \%)$ & $2 / 5(40 \%)$ \\
\hline Lower limbs & $322 / 499(64.5 \%)$ & $259 / 338(65.2 \%)$ & $10 / 17(58.9 \%)$ & $31 / 42(73.8 \%)$ & $12 / 20(60 \%)$ & $6 / 17(35.3 \%)$ & $3 / 5(60 \%)$ \\
\hline
\end{tabular}

SD), 2 in the upper limbs (mean 1.9 SD 1.2) and 2 in the lower limbs (mean 2.5, SD 1.2).

The univariate logistic regression analysis showed an odds ratio (OR) of 1.59 (CI: $1.40-1.81 ; z: 7.2 ; p<0.00$ ) meaning that for each nerve added to neurophysiological evaluation there was an additional $60 \%$ probability to make an electrophysiological diagnosis of definite or probable CIDP (Fig. 3). Repeating this evaluation for upper and lower limbs separately, upper limbs demonstrated higher value (OR: 1.97; CI: $1.64-2.36 ; z=7.28 ; p<0.00)$ than lower limbs (OR: 1.36; CI: $1.15-1.62 ; z: 3.56 ; p<0.00)$.

In possible CIDP patients as well in those not fulfilling CIDP electrophysiological criteria, proximal TD was calculated less times compared to definite and probable CIDP patients (median value: 0 vs 4 times); $p<0.00$ ).

The logistic regression analysis demonstrated an OR of 1.33 (CI: $1.22-1.45 ; z: 6.52 ; p<0.00$ ), meaning that for each TD measurement, there was an additional $33 \%$ probability to reach an electrophysiological diagnosis of definite or probable CIDP (Fig. 3). To exclude that this finding may simply depend on the greater number of tested motor nerves, we performed a multiple logistic regression analysis using, as independent variables, both the number of tested nerves and TD measurements, and as dependent variable the diagnostic class (CIDP (definite, probable) group vs possible and not-fulfilling criteria group), and we confirmed a significant relationship for both the independent variables ( $p<0.00$ for both variables).

Lastly, regression analysis for the number of tested nerves and for the number of proximal temporal dispersion measurement showed that the study of more than 6 nerves did not further improve the diagnostic rate of definite or probable CIDP (Table 4).

\section{Discussion}

We conducted an extensive analysis on the neurophysiological features in a very large cohort of CIDP patients from the Italian CIDP Database.

Nerve conduction slowing is typically considered the hallmark of demyelinating diseases; thus, it is not surprising that, among all the demyelinating features, MNCV was the most frequently altered (68\% of patients) and showed the best diagnostic accuracy (AUC: 0.8401).

The most informative nerve was the ulnar nerve, which had the highest rate of demyelinating features (right $=67.9 \%$; left= $69.3 \%$ ), as well as ulnar nerve tested bilaterally, which showed the highest diagnostic accuracy in CIDP population as a whole (AUC: 0.84 ) and in each CIDP category except for DADS, in which peroneal nerves followed by tibial nerves were the most informative nerves.

Lower rate of demyelinating features as well as lower diagnostic accuracy (AUC: 0.78 ) of median with nerve respect to ulnar nerve could depend on the exclusion of prolonged DML from our analysis as a possible sign of carpal tunnel syndrome, when coupled with a consistent sensory conduction study. This certainly reduces the diagnostic potential of

Table 3 ROC curve analysis

\begin{tabular}{|c|c|c|c|c|c|c|c|}
\hline & $\begin{array}{l}\text { Per nerve } \\
\text { (overall) }\end{array}$ & $\begin{array}{l}\text { Per couple } \\
\text { (overall) }\end{array}$ & $\begin{array}{l}\text { Per couple } \\
\text { (typical) }\end{array}$ & $\begin{array}{l}\text { Per couple } \\
\text { (MADSAM) }\end{array}$ & $\begin{array}{l}\text { Per couple } \\
\text { (DADS) }\end{array}$ & $\begin{array}{l}\text { Per couple } \\
\text { (sensory) }\end{array}$ & $\begin{array}{l}\text { Per couple } \\
\text { (motor) }\end{array}$ \\
\hline $\begin{array}{l}\text { Right median } \\
\text { nerve }\end{array}$ & 0.7133 & 0.7880 & 0.8019 & 0.6894 & 0.6833 & 0.8 & 0.9 \\
\hline $\begin{array}{l}\text { Left median } \\
\text { nerve }\end{array}$ & 0.7092 & & & & & & \\
\hline Right ulnar nerve & 0.7530 & 0.8401 & 0.8509 & 0.9167 & 0.7347 & 0.92 & 0.9157 \\
\hline Left ulnar nerve & 0.7396 & & & & & & \\
\hline $\begin{array}{l}\text { Right peroneal } \\
\text { nerve }\end{array}$ & 0.7045 & 0.7795 & 0.7719 & 0.5758 & 0.9167 & 0.84 & 0.7 \\
\hline $\begin{array}{l}\text { Left peroneal } \\
\text { nerve }\end{array}$ & 0.7185 & & & & & & \\
\hline Right tibial nerve & 0.7066 & 0.7577 & 0.7499 & 0.6667 & 0.8625 & 0.825 & 0.6786 \\
\hline Left tibial nerve & 0.6929 & & & & & & \\
\hline
\end{tabular}


Fig. 1 ROC curve analysis showing diagnostic accuracy for each nerve in the entire population

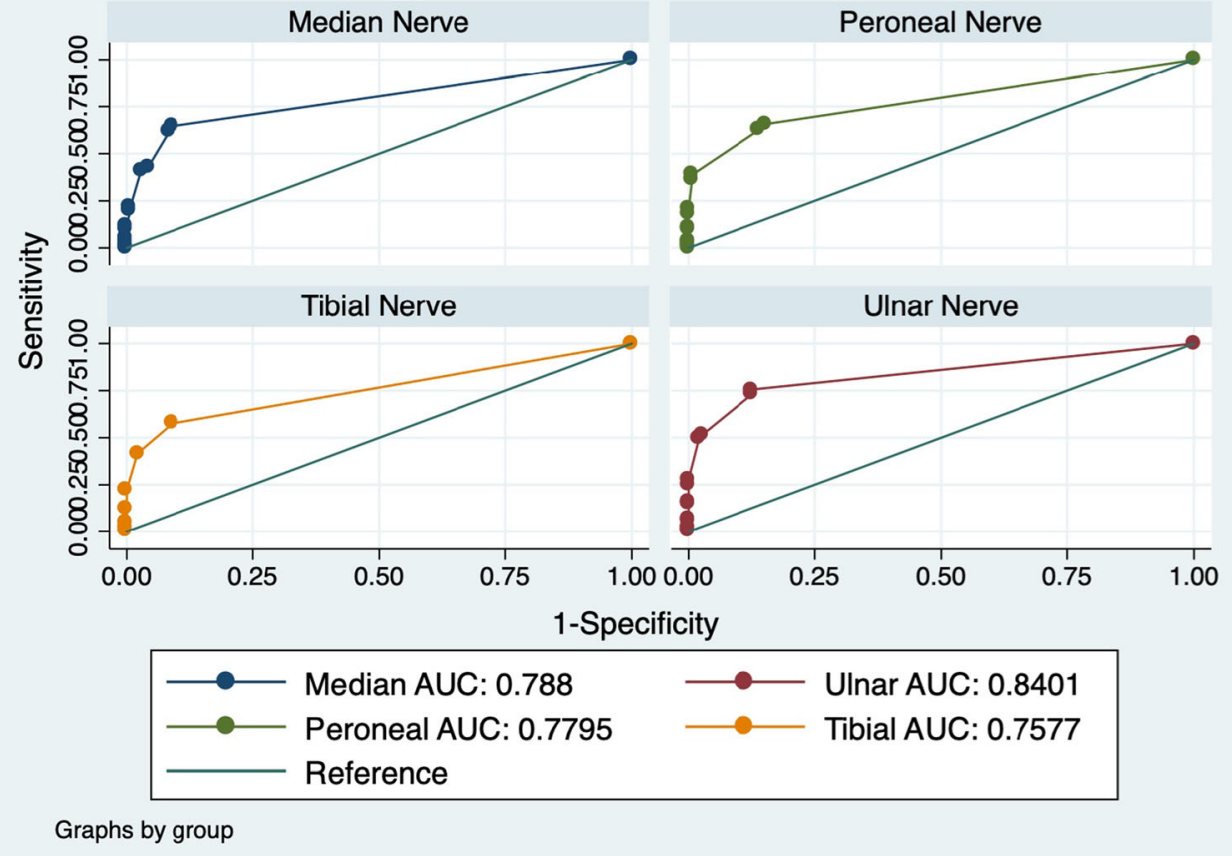

the median nerve and it should be properly considered when performing the electrophysiological exam.

On the other hand, the fact that the lower limb nerves had the most diagnostic accuracy in DADS patients is certainly consistent with a length-dependent neuropathy such as DADS neuropathy.
When facing a suspected CIDP patient, it is well known that if electrophysiological criteria for demyelination are not satisfied, the diagnosis as well as the access to treatment may be delayed or denied. Thus, in order not to miss early diagnosis of CIDP, several diagnostic criteria have been proposed and the most widely accepted criteria are those recommended
Fig. 2 ROC curve analysis showing diagnostic accuracy for each demyelinating feature in the entire population

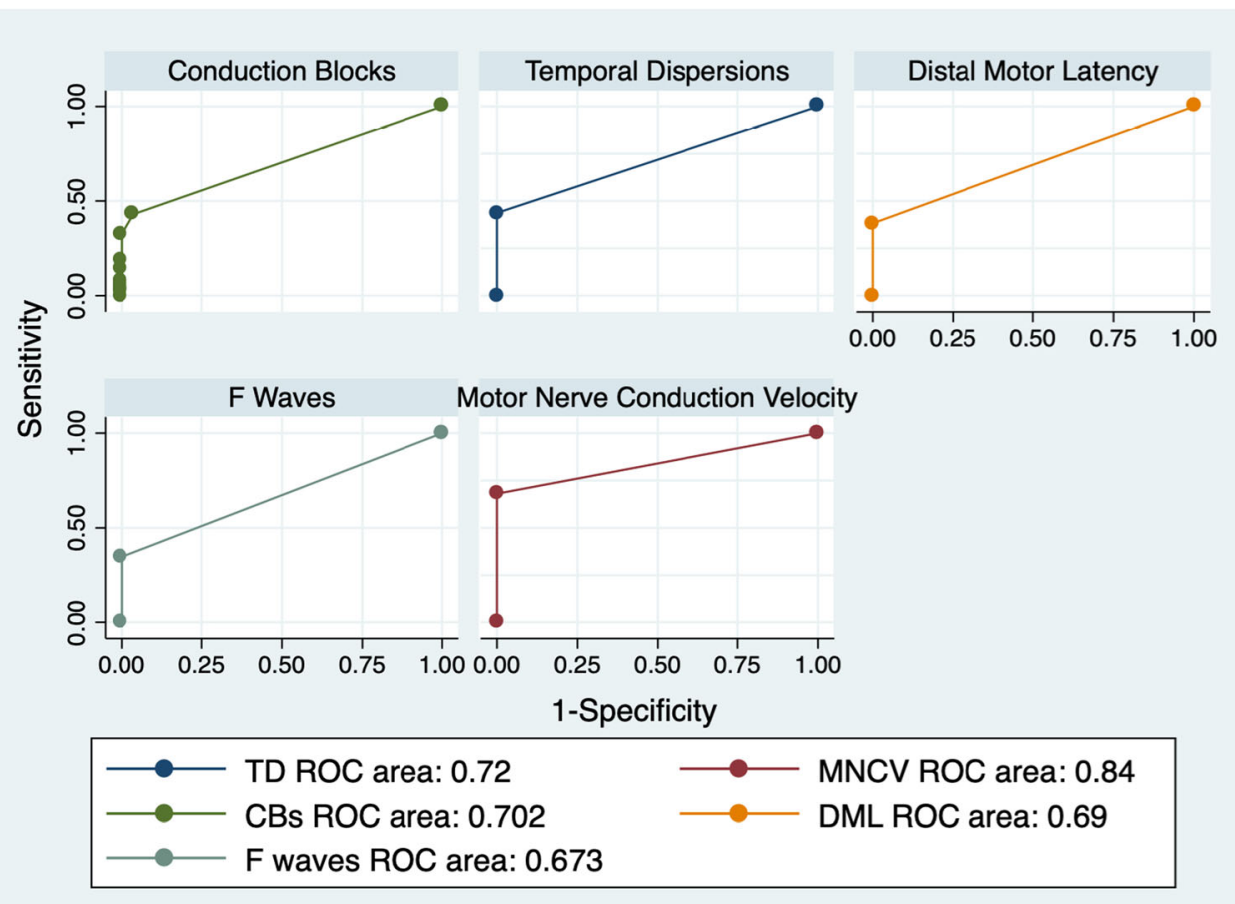


Fig. 3 Diagnostic odds ratio for each tested nerve and proximal temporal dispersion measurement

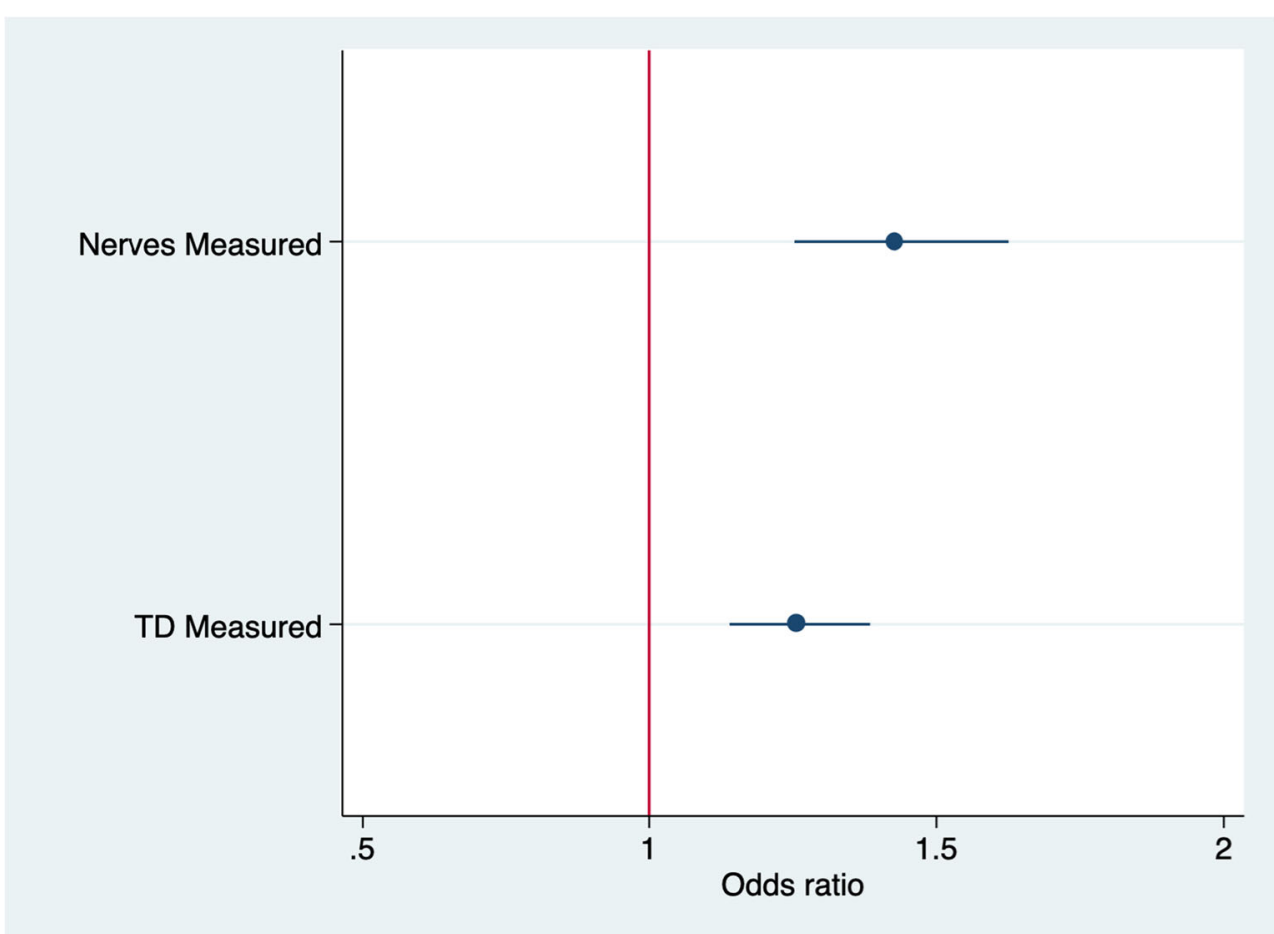

by EFNS/PNS which appear to have the best combination of sensitivity $(73 \%)$ and specificity $(90 \%)$ for the diagnosis of CIDP [5-7]. Importantly, our $16 \%$ rate of patients not fulfilling the EFNS/PNS criteria is fully in line with sensitivity of applied criteria [6, 14]. Anyway, these patients have been considered affected by CIDP because of their clinical features together with supportive EFNS/PNS criteria. Indeed, we discussed clinical and laboratory features and we reconsidered a list of an alternative diagnosis, and collectively we reached a diagnostic consensus to minimize the possibility of a misdiagnosis [15-19].

Although expected, our analysis certainly demonstrated that the diagnosis of CIDP may be missed due to inadequate or incomplete electrophysiological examination or interpretation. Indeed, in possible and not-fulfilling CIDP patients, a lower number of motor nerves (median value 4 vs 6 ) and of TD (median value: 0 vs 4 ) measurements had been performed compared to definite and probable CIDP patients.
Consistently, the calculation of odds ratio revealed that each nerve added to examination increased the probability (OR: 1.59) to reach an electrophysiological diagnosis of definite and probable CIDP, especially when nerves of the upper limbs were tested (OR: 1.97). However, the investigation of more than 6 nerves did not improve further the diagnostic rate of CIDP.

In addition, for each proximal TD measurement, there was an additional $33 \%$ probability to reach an electrophysiological diagnosis of definite and probable CIDP, regardless of the number of tested motor nerves.

These data taken together could be useful to draw a thoughtful electrophysiological approach to patients suspected of CIDP. The joint task force of EFNS/PNS guidelines reports to test at first four nerves (median, ulnar, peroneal, and tibial nerves) on one side and, if criteria are not fulfilled, to study the same nerves at the other side.
Table 4 Distribution of percentage of diagnosed patients according to number of tested nerves and statistical comparison

\begin{tabular}{llll}
\hline Tested nerves & Number of patients & Definite + probable diagnosis $(\%)$ & Comparison with cut-off (6) \\
\hline 2 & 26 & $4 / 26(15.4 \%)$ & 6 vs $2 ; p<0.00$ \\
3 & 59 & $26 / 59(44.1 \%)$ & 6 vs $3 ; p<0.00$ \\
4 & 87 & $59 / 87(67.8 \%)$ & 6 vs $4 ; p<0.00$ \\
5 & 65 & $49 / 65(75.4 \%)$ & 6 vs $5 ; p=0.26$ \\
6 & 137 & $114 / 137(83.2 \%)$ & Reference value \\
7 & 49 & $43 / 49(87.7 \%)$ & 6 vs $7 ; p=0.45$ \\
8 & 76 & $61 / 76(80.2 \%)$ & 6 vs $8 ; p=0.59$ \\
Total & 499 & $356 / 499(71.3 \%)$ & \\
\hline
\end{tabular}


Our findings suggest to start electrophysiological examination by testing ulnar and peroneal nerves bilaterally; afterward, taking into account the cut-off of six nerves, adding tibial nerves for DADS phenotype and median nerves for all other CIDP subtypes (typical, MADSAM, sensory and motor) could improve the electrophysiological diagnostic chance. Importantly, electrophysiological examination should consider all nerve conduction demyelinating findings including the evaluation of temporal dispersion.

However, distal CMAP amplitudes $(<1 \mathrm{mV})$ and especially from peroneal and tibial nerves may be too low for properly interpreting MNCV slowing as demyelinating [20, 21]. Moreover, both prolonged minimal F-wave latencies and absent $\mathrm{F}$-waves may be classified as demyelinating features, but these findings are not specific, especially in the case of the peroneal nerve $[20,22]$.

Lastly, we are absolutely aware that distal CMAP duration can be very useful for CIDP diagnosis, but unfortunately, we could not consider it, as, given the retrospective nature of this study, filter settings to adjust the cut-off values were not available $[23,24]$.

Supplementary Information The online version contains supplementary material available at https://doi.org/10.1007/s10072-021-05321-z.

Author contribution ES contributed to the conception of the research project, reviewed and commented on the statistical analysis, wrote the first draft of the report, and reviewed the report. GL, DC, RF, CB, MF, LB, GA, GC, SJ, AM, AC, GAN, AMC, GS, MC, ML, GL, TR, GC, EP, ST, MR, SCP, AS, LL, LG, LP, GM, LS contributed to the conception, organization, and execution of the research project, reviewed and commented on the statistical analysis and the report. ES designed and executed the statistical analysis, contributed to the conception, organization, and execution of the research project, reviewed and commented on the statistical analysis and the report. ENO and FM conceived, organized and designed the study, reviewed and commented on the statistical analysis, wrote the first draft of the report, reviewed the report.

Funding Open access funding provided by Università degli Studi di Napoli Federico II within the CRUI-CARE Agreement.

\section{Declarations}

Conflict of interest P.E.D. reported travel grants to attend scientific meetings from CSL Behring and Kedrion.

G.L. reported travel grants to attend scientific meetings from CSL Behring and Kedrion.

D.C. reported honoraria for lecturing from Shire, CSL Behring, and Kedrion and travel grants to attend scientific meeting from Shire, Kedrion, and CSL Behring.

R.F. has served on scientific advisory boards for CSL Behring and has received travel grants from Kedrion and CSL Behring to attend scientific meeting.

C.B. has served on scientific advisory boards for Pfizer and has received travel grants from Kedrion and CSL Behring to attend scientific meeting.
M.F. has served on scientific advisory boards for CSL Behring and Sarepta Therapeutics and has received travel grants from Sanofi Genzyme, Kedrion, Baxter and CSL Behring to attend scientific meeting.

G.A. reported honoraria for lecturing from Kedrion and Sanofi Genzyme, travel grants from Kedrion, Sanofi-Genzyme, and LJ Pharma.

G.C. reported travel grants to attend scientific meetings from CSL Behring and Kedrion.

S.J. reported research grants from Grifols, outside this work, and travel grants from Grifols and Kedrion.

A.M. reported travel grants from Kedrion and CSL Behring to attend scientific meeting.

A.C. reported travel grants to attend scientific meetings from Kedrion.

G.A.M. reported consulence fees and travel fundings from CSL Behring, Kedrion, Shire, and Grifols.

M.C. reported travel grants to attend scientific meetings from Kedrion.

M.L. reported honoraria for scientific board from Pfizer and Alnylam and travel grants from Pfizer, Grifols, and Kedrion to attend scientific meeting.

G.C. reported honoraria for lecturing and travel grants to attend scientific meetings from Kedrion.

E.P. reported travel grants to attend scientific meetings from CSL Behring.

L.G. reported travel grants from Kedrion and CSL Behring to attend scientific meeting.

G.M. reported consulence fees and travel fundings from CSL Behring, Kedrion, Shire, and Grifols.

L.S. reported personal fees for scientific events from CSL Behring and has received travel grants to attend scientific meetings from CSL Behring and Kedrion.

E.N.O. reported personal fees for Advisory or Scientific Board from Kedrion, Italy; Baxter, Italy; Novartis, Switzerland; CSL Behring, Italy; LFB, France; and Astellas, the Netherlands; outside the submitted work and travel grants to attend Scientific Meeting from Baxter, Grifols, Kedrion, and Novartis, Italy.

F.M. reported personal fees for scientific events from CSL Behring and has received travel grants to attend scientific meetings from CSL Behring and Kedrion.

The other authors declare no conflict of interest.

Ethical approval The Ethic Committee of each participating Center approved the study. All the patients gave written informed consent.

Open Access This article is licensed under a Creative Commons Attribution 4.0 International License, which permits use, sharing, adaptation, distribution and reproduction in any medium or format, as long as you give appropriate credit to the original author(s) and the source, provide a link to the Creative Commons licence, and indicate if changes were made. The images or other third party material in this article are included in the article's Creative Commons licence, unless indicated otherwise in a credit line to the material. If material is not included in the article's Creative Commons licence and your intended use is not permitted by statutory regulation or exceeds the permitted use, you will need to obtain permission directly from the copyright holder. To view a copy of this licence, visit http://creativecommons.org/licenses/by/4.0/.

\section{References}

1. Querol L, Crabtree M, Herepath M, Priedane E, Viejo Viejo I, Agush S, Sommerer P (2020) Systematic literature review of burden of illness in chronic inflammatory demyelinating polyneuropathy (CIDP). J Neurol. https://doi.org/10.1007/s00415020-09998-8 
2. Spina E, Topa A, Iodice R, Tozza S, Ruggiero L, Dubbioso R, Esposito M, Bruzzese D, Santoro L, Manganelli F (2017) Early predictive factors of disability in CIDP. J Neurol 264:1939-1944

3. Doneddu PE, Cocito D, Manganelli F, Fazio R, Briani C, Filosto M, Benedetti L, Mazzeo A, Marfia GA, Cortese A, Fierro B, Jann S, Beghi E, Clerici AM, Carpo M, Schenone A, Luigetti M, Lauria G, Antonini G, Rosso T, Siciliano G, Cavaletti G, Liberatore G, Santoro L, Peci E, Tronci S, Ruiz M, Cotti Piccinelli S, Toscano A, Mataluni G, Piccolo L, Cosentino G, Sabatelli M, Nobile-Orazio E, Italian CIDP Database study group (2019) Atypical CIDP: diagnostic criteria, progression and treatment response. Data from the Italian CIDP Database. J Neurol Neurosurg Psychiatry 90:125-132

4. Doneddu PE, Bianchi E, Cocito D, Manganelli F, Fazio R, Filosto M, Mazzeo A, Cosentino G, Cortese A, Jann S, Clerici AM, Antonini G, Siciliano G, Luigetti M, Marfia GA, Briani C, Lauria G, Rosso T, Cavaletti G, Carpo M, Benedetti L, Beghi E, Liberatore G, Santoro L, Peci E, Tronci S, Cotti Piccinelli S, Toscano A, Piccolo L, Verrengia EP, Leonardi L, Schirinzi E, Mataluni G, Ruiz M, Dacci P, Nobile-Orazio E, Italian CIDP Database Study Group (2020) Risk factors for chronic inflammatory demyelinating polyradiculoneuropathy (CIDP): antecedent events, lifestyle and dietary habits. Data from the Italian CIDP Database. Eur J Neurol 27(1):136-143

5. Rajabally YA, Nicolas G, Piéret F, Bouche P, Van den Bergh PY (2009) Validity of diagnostic criteria for chronic inflammatory demyelinating polyneuropathy: a multicentre European study. J Neurol Neurosurg Psychiatry 80:1364-1368

6. Van den Bergh PY, Hadden RD, Bouche P, Cornblath DR, Hahn A, Illa I et al (2010) European Federation of Neurological Societies/ Peripheral Nerve Society guideline on management of chronic inflammatory demyelinating polyradiculoneuropathy: report of a joint task force of the European Federation of Neurological Societies and the Peripheral nerve society. Eur J Neurol 17:356363

7. Breiner A, Brannagan TH 3rd. (2014) Comparison of sensitivity and specificity among 15 criteria for chronic inflammatory demyelinating polyneuropathy. Muscle Nerve 50:40-46

8. Inaba A, Yokota T, Saito Y, Ichikawa T, Mizusawa H (2001) Proximal motor conduction evaluated by transcranial magnetic stimulation in acquired inflammatory demyelinating neuropathies. Clin Neurophysiol 112:1936-1945

9. Padua L, Granata G, Sabatelli M, Inghilleri M, Lucchetta M, Luigetti M, Coraci D, Martinoli C, Briani C (2014) Heterogeneity of root and nerve ultrasound pattern in CIDP patients. Clin Neurophysiol 125:160-165

10. Shibuya K, Tsuneyama A, Misawa S, Sekiguchi Y, Beppu M, Suichi T, Suzuki YI, Nakamura K, Kano H, Kuwabara S (2020) Different distribution of demyelination in chronic inflammatory demyelinating polyneuropathy subtypes. J Neuroimmunol 341: 577170. https://doi.org/10.1016/j.jneuroim.2020.577170 Epub 2020 Jan 24

11. Liberatore G, Manganelli F, Cocito D, Fazio R, Briani C, Filosto M, Benedetti L, Antonini G, Cosentino G, Jann S, Mazzeo A, Cortese A, Marfia GA, Clerici AM, Siciliano G, Carpo M, Sabatelli M,
Lauria G, Rosso T, Nobile Orazio E (2020) on the behalf of the Italian CIDP Database Study Group. Relevance of diagnostic investigations in chronic inflammatory demyelinating poliradiculoneuropathy: data from the italian cidp database. $J$ Peripher Nerv Syst 28:152-161. https://doi.org/10.1111/jns.12378

12. Mandrekar JN (2010) Receiver operating characteristic curve in diagnostic test assessment. J Thorac Oncol 5:1315-1316

13. Sotgiu S, Minutolo A, Carta A, Puseddu G, Doneddu PE, NobileOrazio E (2021) Chronic immune sensory polyradiculopathy (CISP): first juvenile case description. Neurol Sci 42:333-336. https://doi.org/10.1007/s10072-020-04588-y

14. Uncini A, Aretusi G, Manganelli F, Sekiguchi Y, Magy L, Tozza S, Tsuneyama A, Lefour S, Kuwabara S, Santoro L, Ippoliti L (2020) Electrodiagnostic accuracy in polyneuropathies: supervised learning algorithms as a tool for practitioners. Neurol Sci 41:3719-3727. https://doi.org/10.1007/s10072-020-04499-y

15. Allen JA, Lewis RA (2015) CIDP diagnostic pitfalls and perception of treatment benefit. Neurology 85:498-504

16. Ricci L, Luigetti M, Florio M et al (2019) Cause of chronic neuropathies: a single-centre experience. Neurol Sci 40:1611-1617. https://doi.org/10.1007/s10072-0129-03899-z

17. Allen JA (2020) The Misdiagnosis of CIDP: A Review. Neurol Ther 9:43-54

18. Allen JA, Ney J, Lewis RA (2018) Electrodiagnostic errors contribute to chronic inflammatory demyelinating polyneuropathy misdiagnosis. Muscle Nerve 57:542-549

19. Gorson KC (2018) Electrodiagnostic misdiagnosis of chronic inflammatory demyelinating polyneuropathy: what are the problems? and how to right the wrongs. Muscle Nerve 57:517-519

20. Eftimov F, Lucke IM, Querol LA, Rajabally YA, Verhamme C (2020) Diagnostic challenges in chronic inflammatory demyelinating polyradiculoneuropathy. Brain 143:3214-3224

21. Van Asseldonk JTH, Van den Berg LH, Kalmijn S, Wokke JHJ, Franssen H (2005) Criteria for demyelination based on the maximum slowing due to axonal degeneration, determined after warming in water at 37 degrees $\mathrm{C}$ : diagnostic yield in chronic inflammatory demyelinating polyneuropathy. Brain 128:880-891

22. Rajabally YA, Varanasi S (2013) Practical electrodiagnostic value of F-wave studies in chronic inflammatory demyelinating polyneuropathy. Clin Neurophysiol 124:171-175

23. Rajabally YA, Lagarde J, Cassereau J, Viala K, Fournier E, Nicolas G (2012) A European multicentre reappraisal of distal compound muscle action potential duration in chronic inflammatory demyelinating polyneuropathy. Eur J Neurol 19(4):638-642

24. Mitsuma S, Van den Bergh P, Rajabally YA, Van Parijs V, MartinLamb D, Sonoo M, Inaba A, Shimizu T, Isose S, Sato Y, Komori T, Misawa S, Kuwabara S, Tokyo Metropolitan Neuromuscular Electrodiagnosis Study Group (2015) Effects of low frequency filtering on distal compound muscle action potential duration for diagnosis of CIDP: a Japanese-European multicenter prospective study. Clin Neurophysiol 126:1805-1810

Publisher's note Springer Nature remains neutral with regard to jurisdictional claims in published maps and institutional affiliations. 


\section{Affiliations}

Emanuele Spina ${ }^{1}$ (D) $\cdot$ Pietro Emiliano Doneddu ${ }^{2} \cdot$ Giuseppe Liberatore $^{2} \cdot$ Dario Cocito $^{3} \cdot$ Raffaella Fazio $^{4} \cdot$ Chiara Briani ${ }^{5}$ - Massimiliano Filosto ${ }^{6} \cdot$ Luana Benedetti $^{7,8} \cdot$ Giovanni Antonini $^{9}$ - Giuseppe Cosentino ${ }^{10,11}$. Stefano Jann ${ }^{12}$. Anna Mazzeo ${ }^{13}$. Andrea Cortese ${ }^{11,14} \cdot$ Girolama Alessandra Marfia ${ }^{15}$. Angelo Maurizio Clerici ${ }^{16}$. Gabriele Siciliano ${ }^{17}$. Marinella Carpo ${ }^{18} \cdot$ Marco Luigetti $^{19}$. Giuseppe Lauria ${ }^{20,21} \cdot$ Tiziana Rosso $^{22} \cdot$ Guido Cavaletti $^{23}$. Erdita Peci ${ }^{3}$. Stefano Tronci ${ }^{4}$ - Marta Ruiz ${ }^{5}$ - Stefano Cotti Piccinelli ${ }^{6}$ - Angelo Schenone ${ }^{7}$ - Luca Leonardi ${ }^{9}$. Luca Gentile $^{13}$. Laura Piccolo $^{11}$. Giorgia Mataluni ${ }^{15}$ • Lucio Santoro ${ }^{1}$ • Eduardo Nobile-Orazio ${ }^{2,24}$ • Fiore Manganelli $^{1}$. on the behalf of the Italian CIDP Database Study Group

1 Department of Neuroscience, Reproductive Sciences and Odontostomatology, University of Naples 'Federico II', Via Pansini, 5, 81025 Naples, Italy

2 Neuromuscular and Neuroimmunology Service, Humanitas Clinical and Research Institute, Rozzano, Milan, Italy

3 Presidio Sanitario Major, Istituti Clinici Scientifici Maugeri, Turin, Italy

4 Division of Neuroscience, Department of Neurology, Institute of Experimental Neurology (INSPE), San Raffaele Scientific Institute, Milan, Italy

5 Neurology Unit, Department of Neuroscience, University of Padova, Padova, Italy

6 Center for Neuromuscular Diseases and Neuropathies, Unit of Neurology, ASST 'Spedali Civili', University of Brescia, Brescia, Italy

7 Department of Neuroscience, Rehabilitation, Ophthalmology, Genetics, Maternal and Child Health, University of Genoa and IRCCS San Martino, Genoa, Italy

8 IRCCS AOU San Martino-IST, Genoa, Italy

9 Unit of Neuromuscular Diseases, Department of Neurology Mental Health and Sensory Organs (NESMOS), Faculty of Medicine and Psychology, 'Sapienza' University of Rome, Sant'Andrea Hospital, Rome, Italy

10 Department of Brain and Behavioral Sciences, University of Pavia, Pavia, Italy

11 IRCCS Foundation C. Mondino National Neurological Institute, Pavia, Italy
12 Department of Neuroscience, Niguarda Ca' Granda Hospital, Milan, Italy

13 Department of Clinical and Experimental Medicine, Unit of Neurology, University of Messina, Messina, Italy

14 Molecular Neurosciences, University College London, London, UK

15 Dysimmune Neuropathies Unit, Department of Systems Medicine, Tor Vergata University of Rome, Rome, Italy

16 Neurology Unit, Circolo \& Macchi Foundation Hospital, Insubria University, DBSV, Varese, Italy

17 Neurology Unit, Department of Clinical and Experimental Medicine, University of Pisa, Pisa, Italy

18 Neurology Unit, ASST Bergamo Ovest-Ospedale Treviglio, Treviglio, Italy

19 Fondazione Policlinico Universitario A. Gemelli IRCCS, UOC Neurologia, Università Cattolica del Sacro Cuore, Rome, Italy

20 Unit of Neuroalgology, IRCCS Foundation 'Carlo Besta' Neurological Institute, Milan, Italy

21 Department of Biomedical and Clinical Sciences 'Luigi Sacco', University of Milan, Milan, Italy

22 ULSS2 Marca Trevigiana, UOC Neurologia-Castelfranco Veneto, Treviso, Italy

23 School of Medicine and Surgery and Experimental Neurology Unit, University of Milano-Bicocca, Monza, Italy

24 Department of Medical Biotechnology and Translational Medicine, Milan University, Milan, Italy 